\title{
$\rightarrow$ \\ Mutaciones del gen HFE y riesgo de carcinoma hepatocelular
}

En 1996, Feder y cols. $(1,2)$ comunicaron el hallazgo de un gen en el brazo corto del cromosoma 6 (6p21.3), próximo a la región del HLA, que posteriormente pasó a denominarse HFE y que se relacionaba con la hemocromatosis hereditaria $(\mathrm{HH})$. Este gen codifica una glicoproteína de 343 aminoácidos -perteneciente a la familia de las moléculas de histocompatibilidad de clase I- que se une a la $\beta 2$-microglobulina y se expresa en la membrana de algunas células. Esta proteína interacciona en la membrana celular con el receptor de la transferrina 1 (TfR1), reduce la afinidad de esta por la transferrina y, en consecuencia, interviene en el metabolismo del hierro $(3,4)$. En ella se pueden reconocer tres asas extracelulares $\left(\alpha_{1}, \alpha_{2}\right.$ y $\left.\alpha_{3}\right)$ y una región corta intracelular. Su interacción con el TfR-1 se realiza a través de una depresión existente entre las asas $\alpha_{1}$ y $\alpha_{2}$ (5). El asa $\alpha_{3}$ se forma por existir un puente tiónico entre las dos cisteínas. Esta asa juega una función esencial para su unión no-covalente a la $\beta_{2}$-microglobulina $\left(\beta_{2} \mathrm{MG}\right)$ y para junto a esta su transporte desde el retículo endoplásmico donde se sintetiza a la superficie de las células (6). En la publicación original de Feder y cols. ya se señalaba que existían dos mutaciones en ese gen cuya presencia se encontraba estrechamente relacionada con la HH. Estas dos mutaciones eran la $845 \mathrm{G} \rightarrow \mathrm{A}(\mathrm{C} 282 \mathrm{Y})$ y la sustitución $187 \mathrm{G} \rightarrow \mathrm{C}(\mathrm{H} 63 \mathrm{D})$. La primera impide la unión tiólica que da lugar a la formación del asa $\alpha_{3}$ de la molécula HFE. Por esta razón, la molécula HFE que se sintetiza en el retículo endoplásmico no puede unirse a la $\beta_{2} \mathrm{MG}$ y, en consecuencia, no puede migrar a la membrana celular y no se aparece en la superficie de las células (3). La segunda mutación, la $187 \mathrm{G} \rightarrow \mathrm{C}$, origina el cambio de una histidina por un aspartato en el aminoácido de la posición 63 (H63D). Este cambio es menos trascendente, ya que no impide que la HFE se localice en las membranas celulares y que interaccione con el TfR 1 . Sin embargo, es posible que esta mutación disminuya la afinidad de la molécula HFE por su ligando, el TfR-1 y, con ello, favorezca al absorción intestinal de hierro (2). Más recientemente se han descrito otras muchas mutaciones en el gen HFE cuya significación clínica es dudosa (7). La más frecuente se localiza en la vecindad de la H63D, es la S65C, que también parece ejercer efectos sobre el metabolismo del hierro, ya que su presencia se ha asociado con cierto grado de siderosis, en especial cuando al mismo tiempo está presente la $\mathrm{C} 282 \mathrm{Y}$, existe abuso alcohólico o algún otro factor que favorezca la sobrecarga de hierro (8).

En su primera publicación sobre el gen de la HFE, Feder y cols. destacaron que estas dos mutaciones se encontraban estrechamente relacionadas con la acumulación excesiva de hierro en el organismo y con la existencia de una HH (1). Esta relación ha sido confirmada por numerosos estudios posteriores, si bien la frecuencia de esta asociación varía mucho de unos países a otros. Mientras que es alta en los países del norte de Europa, es más baja en los de la cuenca mediterránea y excepcional 
en regiones habitadas por población de origen no europeo. España, sin duda país mediterráneo, se diferencia de lo que ocurre en otros países del sur de Europa. Mientras que en Grecia, Italia y sur de Francia, el 30, 64,5 y 72,3\%, respectivamente, de las HH son homocigotos C282Y, en España esto ocurre en el 87\% (9). Esta frecuencia nos acerca a lo que ocurre en los países del norte de Europa, por ejemplo, a Alemania (94,6\%), Reino Unido (91,3\%), Suecia (92\%), Irlanda (93\%) o Bretaña (96\%) (10). Una excepción a este comportamiento genético es la población vasca española o francesa, donde la $\mathrm{HH}$ se encuentra justificada por la mutación C282Y en el 57,1 y 53,3\%, respectivamente, de los casos. Algunos enfermos con $\mathrm{HH}$, su trastorno metabólico se explica por presentar la doble mutación heterocigota C282Y/H63D. Esto ocurre en el 6,5\% de la población española y en el 11,4 y 13,3\% de la población vasca española y francesa, respectivamente $(11,12)$. Esta peculiar distribución geográfica de la HH ligada a la mutación C282Y se explica fácilmente por su relación con las migraciones del pueblo celta. Este, de origen indo-europeo, se asentó a finales de la edad de bronce en el centro de Europa y desde aquí se extendió hacia el norte de Europa, pero también por la Península Ibérica, Francia, Suiza, Islas Británicas, etc. (13). Probablemente, el País Vasco quedó al margen de esta migración. El momento en el que se produjo la mutación C282Y en una familia celta no está bien definido. Para unos se produjo en un momento relativamente reciente, hace tan sólo unas 70 generaciones, 300 años antes de C (14), es decir, después de que se produjera la migración celta. Esto significa, que la llegada de esta mutación a nuestro país y a otros países europeos debió ocurrir en épocas posteriores, quizá con las invasiones del pueblo vikingo. Para otros, la mutación es más antigua y se remontaría a 113 generaciones y, por tanto, su difusión pudo ser paralela a las del pueblo celta (13).

Entre las consecuencias de la sobrecarga de hierro destaca el elevado riesgo de desarrollar un carcinoma hepatocelular (CHC), que en los pacientes con $\mathrm{HH}$ es 19 veces mayor al existente en la población general. En efecto, el $45 \%$ de los pacientes con HH se complican con este tumor hepático (15-17). Es cierto que en la mayoría de los casos, la enfermedad hepática se encuentra en una fase avanzada de su evolución y existe ya una cirrosis hepática: sin embargo, el CHC también se ha descrito en pacientes en quienes aún no existía una cirrosis $(18,19)$. Se supone que el depósito de hierro, quizá por su papel en la formación de sustancias reactivas derivadas del oxígeno (ROS) $(20,21)$, pudiera participar en la mutagénesis que determina la aparición del tumor. A favor de que el depósito de hierro en los tejidos juegue un papel en la carcinogénesis aboga el hecho de que la depleción de hierro en pacientes con $\mathrm{HH}$ sin cirrosis previene la aparición del CHC: en las siderosis hepáticas diferentes a las relacionadas con el HFE, también existe una frecuencia elevada de CHC (22) y, por último, en modelos animales de siderosis hepática también se hallan focos de CHC en ausencia de cirrosis (23). Por la relación existente entre la acumulación excesiva de hierro en los tejidos y las mutaciones del gen HFE, se pudiera esperar que en los pacientes con CHC estas mutaciones fueran frecuentes, en especial, entre los pacientes con acumulación patológica de hierro en los tejidos.

En el presente número de la Revista Española de Enfermedades Digestivas, Ropero y cols. comunican que entre 196 pacientes con $\mathrm{CHC}$ la frecuencia de la mutación $\mathrm{C} 282 \mathrm{Y}$ era similar a la hallada en donantes de sangre sanos españoles de raza blanca. Por el contrario, la frecuencia alélica $(p=0,004)$ y de portadores de la mutación H63D ( $\mathrm{p}=0,002)$ estaba significativamente elevada en los pacientes con CHC (24). Es de destacar que esta asociación no ha sido observada previamente por otros autores. La mayoría de estudios publicados que establecen una relación entre el gen 
HFE y el CHC lo hacen a través de la mutación C282Y. Este es el caso del estudio de Wallis y cols. en el que hallan esta mutación en el 7\% de los enfermos con CHC, mientras que en su población control la hallan sólo en el 0,7\% $(25,26)$. También Lauret y cols. encontraron que la frecuencia de la mutación C282Y, pero no la de la H63D, era significativamente más alta en los pacientes con cirrosis alcohólica y $\mathrm{CHC}$ que en los que carecían de este tumor (27). En el mismo sentido señala el estudio de Hellerbrand y cols., cuando entre 137 pacientes con $\mathrm{CHC}$ sin $\mathrm{HH}$ encuentran que el 12,4\% eran heterocigotos C282Y, mientras que esto ocurría sólo en 3,7\% de los cirróticos sin CHC y en el 4,8\% de los controles sanos. Los autores destacan que esta asociación tenía como base la presencia de sobrecargas anormales de hierro en el organismo. Tampoco en este estudio se encuentra que la frecuencia de la H63D estuviera aumentada en los cirróticos con CHC (28). Por su parte, Aldersley y cols. encontraron la mutación C282Y homocigota o heterocigota en el 6,6 y en el 25\%, respectivamente, de los pacientes con CHC. Prevalencias estas muy superiores al 0 y $5 \%$ encontradas en los controles (29). En contra de lo hallado por los autores referidos, otros aportan resultados discrepantes. Este es el caso de Boige y cols. que estudiaron de forma prospectiva la presencia de mutaciones en el gen HFE en 233 pacientes con cirrosis hepática, 133 con $\mathrm{CHC}$ y 100 sin $\mathrm{CHC}$. Al igual que en el estudio comentado, estos autores no pudieron hallar que en los pacientes con $\mathrm{CHC}$ la prevalencia de la mutación $\mathrm{C} 282 \mathrm{Y}$ fuera diferente a la encontrada en los pacientes sin CHC o en la población general. En contra de lo hallado por Ropero y cols. en el presente número de la revista, la frecuencia de la mutación H63D fue similar en ambos grupos de cirróticos, tuvieran o no la complicación tumoral (30). Cauza y cols., en 162 pacientes con CHC, estudiaron la frecuencia de las dos principales mutaciones asociadas a la $\mathrm{HH}$ y encontraron que si bien el riesgo de padecer un $\mathrm{CHC}$ era 19 veces superior en los homocigotos para la mutación C282Y, los que presentan la mutación H63D no presentan riesgo elevado de desarrollar esta complicación tumoral (31). El valor de este estudio, sin embargo, disminuye si se considera que carece de grupo control adecuado y que los resultados fueron comparados con los publicados previamente por otros para la población austriaca. A la misma conclusión llegaron Racchi y cols. (32), sin embargo, tampoco podemos considerar definitivas estas conclusiones, ya que su serie incluía únicamente 12 pacientes con CHC. Como se puede comprobar existen numerosas discrepancias sobre la frecuencia de las mutaciones del gen HFE en las pacientes con CHC. Es muy probable que ello esté justificado por la naturaleza de la población estudiada, el país donde se realizaron los diferentes estudios, el carácter retrospectivo de algunos de estos, la existencia de factores asociados (alcohol, infecciones virales), del empleo o no de controles cirróticos sin $\mathrm{CHC}$ o de población sana y de número de pacientes incluidos en cada estudio. Sin embargo, todos ello tienen en común que la mutación H63D no parecía estar asociada con la presencia de un CHC. Tan sólo en la publicación de Fracanzani y cols. se menciona que la frecuencia de esta última mutación estaba aumentada en las mujeres cirróticas con CHC infectadas por el virus de la hepatitis C (33). La razón de la asociación hallada por Ropero y cols. es desconocida. El estudio descarta que ello pudiera deberse a la coincidencia de otros factores asociados, tales como las infecciones virales o el alcohol. Tampoco es probable que ello se deba a una sobrecarga patológica de hierro. Aunque los autores no han podido informar sobre la cuantía de hierro existente en el hígado de los pacientes con $\mathrm{CHC}$, ya que el único tejido hepático disponible era el tumoral obtenido por punción con aguja fina, es muy probable que la siderosis, de existir, no fuera particularmente alta. Mientras que la mutación homocigota $\mathrm{C} 282 \mathrm{Y}$ se asocia con una sobrecarga bioquímica de 
hierro (aumento de la saturación de la transferrina, aumento de ferritina sérica) en el $60-70 \%$ de los casos $(34,35)$, la mutación H63D, aún en estado homocigoto, tiene escaso impacto clínico (36) y sólo algún estudio lo ha relacionado con aumento de la saturación de transferrina (37). Tan sólo, cuando coincide con la mutación C282Y, en forma de doble heterocigoto, puede originar siderosis hepática importante y cuadros de $\mathrm{HH}$ (38). Por todo ello es muy probable que la asociación detectada por Ropero y cols. entre el H63D y el CHC no esté determinada por un exceso de hierro en el hígado.

Una posibilidad pudiera ser la existencia en el cromosoma 6 de un área de susceptibilidad tumoral que estuviera ligada genéticamente al locus HFE. Esta eventualidad ha sido mencionada para el cáncer de próstata (39) y pudiera explicar algunas observaciones publicadas por las que los pacientes con mutaciones en el gen HFE tienen un mayor riesgo de presentar un cáncer extrahepático (40). Este sería el caso del exceso de cánceres de mama hallado entre mujeres portadoras de la mutación C282Y (41) o de la H63D (42). Igualmente se ha mencionado que los heterocigotos HFE tienen un riesgo elevado para presentar gliomas malignos (43) o cánceres de colon o estómago. (44). Sin embargo, el papel de estas mutaciones y su asociación con los procesos tumorales no ha podido ser demostrado por estudios diseñados específicamente para resolver este tema $(45,46)$.

Los autores del trabajo comentado sugieren que el parentesco existente entre la molécula HFE y las de histocompatibilidad pudiera influir en la respuesta inmune y, por caminos aún pendientes de investigar, pudiera conducir al desarrollo tumoral. Se trata sin duda de una hipótesis atractiva que deberá ser tenida en consideración en futuros estudios.

Desde hace décadas se viene considerando que la cirrosis hepática, por sí misma, con independencia de su causa, es un factor de riesgo para la aparición de un CHC. En su artículo, Ropero y cols. no especifican claramente la enfermedad hepática de fondo sobre la que asentaba el CHC, sin embargo, lo más probable es que en la mayoría de sus enfermos existiera una cirrosis hepática. En nuestra experiencia, el 78,5\% de los pacientes con CHC presentan una cirrosis hepática y el 13\% de los pacientes diagnosticados laparoscópicamente de cirrosis padecían un CHC. En otras series de pacientes con CHC publicadas en los últimos años en España, la frecuencia de cirrosis en pacientes con este tumor era aún mayor y superaba el 95\% (47,48). Esta transformación maligna de los hepatocitos se piensa que se produce en el contexto de un "turnover" celular acelerado originado por la muerte y regeneración celular, la inflamación crónica, la activación de factores de crecimiento y angiogénicos, el daño del ADN no adecuadamente reparado, la reordenación genética, la activación de oncogenes y de telomerasas y la inactivación de genes supresores $(49,50)$. Factores etiológicos de agresión hepatocelular, tales como la infección crónica por los virus de la hepatitis B y C, el abuso crónico de alcohol y las enfermedades metabólicas -hemocromatosis, deficiencia de alfa-1 antitripsina, tirosinemia, glucogenosis- estarían relacionadas con el CHC a través de este mecanismo. Sin embargo, algunos de estos agentes etiológicos probablemente influyen también en la cancerogénesis por mecanismos más directos. Así, por ejemplo, el virus de la hepatitis B (VHB) puede integrarse en el genoma de las células hepáticas infectadas y puede promover la degeneración maligna de los hepatocitos, ya que al intercalarse en algunos genes, obliga al reordenamiento cromosómico y ello provoca inestabilidad genómica $(51,52)$. Esta integración se ha encontrado en el $80-90 \%$ de los HCC (53). Además, la proteína HBx del VHB probablemente está también implicada en la carcinogénesis hepática, aunque no existe unanimidad sobre los mecanismos por los que actúa (54). 
La infección crónica por el virus de la hepatitis C (VHC) es otra causa importante y creciente de CHC en Europa, Estados Unidos y Japón (55). En los países de nuestro entorno es la causa principal de cirrosis complicada con CHC. Por ejemplo, en un estudio realizado en Valencia y en otro que lo fue en Cantabria, esta infección estaba presente en el 75 y en el $60,6 \%$ de los enfermos, respectivamente $(48,56)$. El riesgo de HCC aumenta aún más cuando la infección por el VHC coincide con la infección por el VHB (50). Los mecanismos por los que el VHC interviene en la carcinogénesis hepática son aún peor conocidos, pero sin duda son diferentes a los que participan cuando la enfermedad se debe al VHB, ya que el genoma del VHC no se integra en el genoma celular. Considerando que en estos tumores existe un marcado aumento de la expresión de genes relacionados con las enzimas de la fase I de la biotransformación de xenobióticos (57), la infección por el VHC pudiera sensibilizar al hígado al efecto de procarcinógenos y carcinógenos, por ejemplo, al etanol. En estos tumores, además, se han encontrado numerosas alteraciones que pudieran intervenir en la génesis tumoral. Así, por ejemplo, se ha descrito que es frecuente el hallazgo de mutaciones en el gen de la $\beta$-catenina (58), reducción del p21, quizá como consecuencia de la represión ejercida por la proteína "c" del VHC y variedades hipoactivas del p53 $(59,60)$. Además, la proteína NS5A del VHC se comporta como antiapoptótica por activar la vía de la fosfatidilinositol 3 quinasa (PI3K) y en consecuencia por permitir la supervivencia de células genéticamente anormales $(61,62)$. Aunque en el estudio de Ropero y cols. no está claramente especificada la causa de la enfermedad hepática de fondo -presumiblemente una cirrosis- parece que un gran número de enfermos padecía una infección crónica viral y un número no determinado probablemente abusaba del alcohol. Por ello, sería interesante conocer, mediante un análisis multivariante, además del papel de las mutaciones del HFE, la contribución que al desarrollo del CHC hayan podido hacer algunos factores coincidentes tales como la cirrosis hepática, las infecciones por los virus B y C de la hepatitis y los hábitos alcohólicos de los pacientes. Muy ilustrativo pudiera ser también, para destacar la asociación del CHC con el H63D, la inclusión de un grupo control formado por cirróticos similares sin evidencias de CHC.

\section{J. A. Solís Herruzo y P. Solís Muñoz}

Servicio de Aparato Digestivo. Hospital Universitario 12 de Octubre. Madrid

\section{Bibliografía}

1. Feder JN, Gnirke A, Thomas W, Tsuchihashi Z, Ruddy DA, Basava A, et al. A novel MHC class I-like gene is mutated in patients with hereditary haemochromatosis. Nat Genet 1996; 13: 399-408.

2. Feder JN, Penny DM, Irrinki A, Lee VK, Lebrón JA, Watson N, et al. The hemochromatosis gene product complexes with the transferrin receptor and lowers its affinity for ligand binding. Proc Natl Acad Sci. USA. 1998; 95: 1472-7.

3. Trinder D, Fox C, Vautier G, Olynyk JK. Molecular pathogenesis of iron overload. Gut 2002; 51: 290-5.

4. Lebron JA, Bennet MJ, Vaughan DE, Chirino AJ, Show PM, Mintier GA, et al. Crystal structure of the hemochromatosis protein HFE and characterization of its interaction with transferrin receptor. Cell 1998; 93: $111-23$.

5. Bennet MJ, Lebron JA, Bjorkman PJ. Crystal structure of the haemochromatosis protein HFE complexed with transferrin receptor. Nature 2000; 403: 46-53.

6. Ehrlich R, Lemonnier FA. HFE: a novel nonclassic class I molecule that is involved in iron metabolism. Immunity 2000; 13: 585-8.

7. Solís-Herruzo JA, Solís-Muñoz P. Non-HFE hemochromatosis. Rev Esp Enferm Dig 2005; 97: 266-86.

8. Wallace DF, Walter AP, Pietrangelo A, Clare M, Bomford AB, Dixon JL, et al. Frequency of the S65C mutation of HFE and iron overload in 309 subjects heterozygous for C282Y. J Hepatol 2002; 36: 474-9. 
9. Sánchez M, Bruguera M, Bosch J, Rodés J, Ballesta F, Oliva R. Prevalence of the Cys282Tyr and His63Asp HFE gene mutations in Spanish patients with hereditary hemochromatosis and in controls. J Hepatol 1998; 29: 725-8.

10. Byrnes V, Ryan E, Barrett S, Kenny P, Mayne P, Crowe J. Genetic hemochromatosis, a celtic disease: is it now time fro population screening? Genetic test 2001; 5: 127-30.

11. De Juan MD, Reta A, Castiella A, Pozueta J, Prado A, Cuadrado E. HFE gene mutations analysis in Basque hereditary haemochromatosis patients and controls. Eur J Hum Genet 2001; 9: 961-4.

12. Bauduer F, Scribans C, Degioanni A, Ronoux M, Dutour O. Distribution of the C282Y and H63D polymorphisms in hereditary hemochromatosis patients from the French Basque Country. Ann Hematol 2005; 84: 99100.

13. Ramalho F. Hemocromatosis hereditaria e influencia de la civilización celta. Disponible en: www.sepd.es.

14. Olsson KR, Ritter D. The ancestral haplotype in patients with hemochromatosis from central and western Sweden. Proce Inter. Symposium on Iron in Biology and Medicine. France, July 1997.

15. Deugnier YM, Guyader D, Crantock L, Lopez JM, Turlin B, Yaouanq J, et al. Primary liver cancer in genetic hemochromatosis: A clinical, pathological, and pathogenetic study of 54 cases. Gastroenterology 1993; 104: 228-34.

16. Francanzani AL, Conte D, Franquelli M, Taioli E, Mattioli M, Losco A, et al. Increased cancer risk in a cohort of 230 patients with hereditary hemochromatosis in comparison to a matched control patients with noniron-related chronic liver disease. Hepatology 2001; 33: 647-51.

17. Cauza E, Peck-Radosavljevic M, Ulrico-Pur H, Datz C, Gschwantle M, Schöniger-Hekele M, et al. Mutations of the HFE gene in patients with hepatocellular carcinoma. Am J Gastroenterol 2003; 98: 442-7.

18. Kew MC. Pathogenesis of hepatocellular carcinoma in hereditary hemochromatosis: occurrence in non-cirrhotic patients. Hepatology 1990; 11: 1086-7.

19. Britto MR, Thomas LA, Balaratnam N, Griffiths AP, Duane PD. Hepatocellular carcinoma in non-cirrhotic liver in genetic haemochromatosis. Scand J Gastroenterol 2000; 35: 889-93.

20. Loeb LA, James EA, Waltersdorph AM, Klebanoff SJ. Mutagenesis by the auto-oxidation of iron with isolated DNA. Proc Natl Acad Sci USA. 1988; 85: 3918-22.

21. Bacon B, Britton RS. The pathology of hepatic iron overload: a free radical-mediated process? Hepatology 1990; 11: 127-37.

22. Mandishona E, McPhail AP, Gordeuk VR, Keedda MA, Peterson AC, Rouault TA, et al. Dietary iron overload as a risk factor for hepatocellular carcinoma in black Africans. Hepatology 1998; 27: 1563-6 .

23. Asare GA, Patterson AC, Kew MC, Kahn S, Mossanda KS. Iron-free neoplastic nodules and hepatocellular carcinoma without cirrhosis in Wistar ratsfed a diet high in iron. J Pathol 2006; 208: 82-90.

24. Ropero P, Briceño O, López-Alonso G, García-Agúndez JA, González-Fernández FA, García-Hoz F, et al. Mutation H63D of the HFE gene is associated with an elevated risk of developing hepatocelular carcinoma. Rev Esp Enferm Dig 2007; 99: 376-81.

25. Wallis G, Wimperis JZ, Lonsdale R, Jennings BA. Haemochromatosis gene mutation in hepatocellular cancer. Lancet 1997; 350: 565-6.

26. Willis G, Bardsley V, Fellows IW, Lonsdale R, Wimperis JZ, Jennings BA. Hepatocellular carcinoma and the penetrance of HFE C282Y mutation: a cross sectional study. BMC Gastroenterol 2005; 5: 17.

27. Lauret E, Rodríguez M, González S, Linares A, López-Vázquez A, Martínez-Borra J, et al. HFE gene mutations in alcoholic and virus-related cirrhotic patients with hepatocelular carcinoma. Am J Gastroenterol 2002; 97: 1016-21.

28. Hellerbrand C, Pöppi A, Hartmann A, Schölmerich J, Lock G. HFE C282Y heterozygosity in hepatocellular carcinoma: evidence for an increased prevalence. Clin Gastroenterol Hepatol 2003; 1: 279-84.

29. Aldersley MA, Komolmit P, Hamlin PJ, et al. Incresed prevalence of the hemochromatosis gene mutation in patients with hepatocellular carcinoma. Gastroenterology 1999; 116: 1182A.

30. Boige V, Castéra L, de Roux N, Ganne-Carrié N, Ducot B, Pelletier G, et al. Lack of association between HFE gene mutation and hepatocellular carcinoma in patients with cirrhosis. Gut 2003; 52: 1178-81.

31. Cauza E, Peck-Radosavijevic M, Ulrich-Pur H, Datz C, Gschwantle M, Schöniger-Hekele M, et al. Mutations of the HFE gene in patients with hepatocellular carcinoma. Am J Gastroenterol 2003; 98: 442-7.

32. Racchi O, Mangerini R, Rapezzi D, Gaetani GF, Nobile MT, Picciotto A, et al. Mutations of the HFE gene and the risk of hepatocellular carcinoma. Blood Cells Mol Dis 1999; 25: 350-3.

33. Francanzani AL, Fargion S, Stazi MA, Valenti L, Amoroso P, Cariani E, et al. Association between heterozygosity for HFE gene mutation and hepatitis virases in hepatocellular carcinoma. Blood Cells Mol Dis 2005; 35: $27-32$.

34. Olynyk JK, Cullen DJ, Aquilia S, Rossi E, Summerville L, Powell LW. A population-based study of the clinical expression of the hemochromatosis gene. N Engl J Med 1999; 341: 718-24.

35. Beutler E, Felitti VJ, Kuziol JA, Ho NJ, Gelbart T. Penetrance of 845G $\rightarrow$ A (C282Y) HFE hereditary hemochromatosis mutation in the USA. Lancet 2002; 359: 211-8.

36. Beutler E. The significance of the 187G (H63D) mutation in hemochromatosis. Am J Hum Genet 1997; 61: $762-4$.

37. Gochee PA, Powell LW, Cullen DJ, Du Sart D, Rossi E, Olynyk JK. A population-based study of the biochemical and clinical expression of the H62D hemochromatosis mutations. Gastroenterology 2002; 122: 64651.

38. Fargion S, Stazi MA, Francanzani AL, Mattioli M, Sampietro M, Tavazzi D, et al. Mutations in the HFE gene and their interaction with exogenous risk factors in hepatocellular carcinoma. Blood Cells Mol Dis 2001; 27: 505-11.

39. Easton DF, Schaid DJ, Whittemore AS, Isaacs WJ. Internacional Consortium for Prostate Cancer Genetics. Where are the prostate cancer genes? A summary of eight genome wide searches. Prostate 2003; 57: 261-9.

40. Geier D, Hebert B, Potti A. Risk of primary non-hepatocellulat malignancies in hereditary hemochromatosis. Anticancer Res 2002; 22: 3797-9. 
41. Kallianpur AR, Hall LD, Yadav M, Christman BW, Dittus RS, Haines JL, et al. Increased prevalence of the HFE C282Y hemochromatosis allele in women with breast cancer. Cancer Epidemiol Biomarkers Prev 2004; 13: 205-12.

42. Barton JC, Bertoli LF, Acton RT. HFE C282Y and H63D in adults with malignancies in a community medical oncology practice. BMC Cancer 2004; 4: 6.

43. Martinez di Montemuros F, Tavazzi D, Salsano E, Piepoli T, Pollo B, Fiorelli G, et al. High frequency of the H63D mutation of the hemochromatosis gene (HFE) in malignant gliomas. Neurology 2001; 57: 1342.

44. Nelson RL, Davis FG, Persky V, Becker E. Risk of neoplastic and other diseases among people with heterozygosity for hereditary hemochromatosis. Cancer 1995; 76: 875-9.

45. Syrjäkosk K, Fredriksson H, Ikonen T, Kuukasjärvi T, Autio V, Matikainen MP, et al. Hemochromatosis gene mutations among Finnish male breast and prostate cancer patients. Int J Cancer 2006; 118: 518-20.

46. Elmberg M, Hultcrantz R, Ekbom A, Brandt L, Olsson S, Olsson R, et al. Cancer risk in patients with hereditary hemochromatosis and in their first-degree relatives. Gastroenterology 2003; 125: 1733-41.

47. Rodríguez-Vidigal FF, Baz MJ, Romero J, Del Puerto M. Epidemiología del carcinoma hepatocelular en un área rural. Papel de los virus hepatotropos en la supervivencia. Ann Intern Med 2005: 22: 162-6.

48. García-Torres ML, Zaragoza A, Giner R, Primo J, Olmo JA. Incidencia y factores epidemiológicos del carcinoma hepatocelular en Valencia durante el año 2000. Rev Esp Enferm Digest 2003; 95: 381-4.

49. Moradpour D, Wands JR. Molecular patogénesis of hepatocellular carcinoma. En: Zakim D, Boyer TD, editors. Hepatology. A textbook of liver disease. $4^{\text {th }}$ ed. Philadelphia: Saunders; 2003. p. 1371-81.

50. Solís Herruzo JA. Hepatocellular carcinoma. Epidemiological and etiological factors. Rev Esp Enferm Dig 2003; 95: 377-80.

51. Rogler CE, Chiasari FV. Cellular and molecular mechanisms of hepatocarcinogenesis. Semin Liver Dis 1992; 12: 265-78

52. Urano Y, Watanabe K, Lin C, Hino O, Tamaoki T. Interstitial chromosomal deletion within 4q11-q13 in a human hepatoma cell line. Cancer Res 1991; 51: 1460-4.

53. Buendía MA Genetics of hepatocellular Carcinoma. Semin Cancer Biol 2000; 10: 185-200.

54. Andrisani OM, Barnabas S. The transcriptional function of the hepatitis B virus X protein and its role in hepatocarcinogenesis. Int J Oncol 1999; 15: 373-9.

55. El-Serag HB, Davila JA, Petersen NJ, McGlynn KA. The continuing increase in the incidence of hepatocellular carcinoma in the United States: an update. Ann Intern Med 2003: 139: 817-23

56. Crespo J, Dueñas C, Sánchez-Antolín G, Fábrega E, Carte B, Pons-Romero F. Infección por el virus de la hepatitis B y C en pacientes con carcinoma hepatocelular. Med Clin (Barc) 1996; 106: 241-5.

57. Okabe H, Satoh S, Kato T, Kitahara O, Yanagawa R, Yamaoka Y, et al. Genome-wide analysis of gene expression in human hepatocellular carcinomas using cDNA microarray. Cancer Res 2001; 61: 2129-37.

58. Hsu HC, Jeng YM, Mao TL, Chu JS, Lai PL, Peng SY. Beta-catenin mutations are associated with a subset of low-stage hepatocellular carcinoma negative for hepatitis B virus and with favorable prognosis. Am J Pathol 2000; 157: 763-70.

59. Anzola M, Cuevas N, López-Martínez M, Saiz A, Burgos JJ, Martínez de Pancorbo M. Frequent loss of p53 codon 72 Pro variant in hepatitis C virus-positive carriers with hepatocellular carcinoma. Cancer Lett 2003; 193: 199-205.

60. Okada F, Shiraki T, Maekawa M, Sato S. A p53 polymorphism associated risk of hepatitis C virus infection. Cancer Lett 2001; 172: 137-42.

61. Street A, Macdonald A, Crowder K, Harris M. The hepatitis C virus NS5A protein activates a phosphoinositide 3-kinase-dependent survival signaling cascade. J Biol Chem 2004; 279: 12232-41.

62. He Y, Nakao H, Tan SL, Polyak PJ, Neddermann P, Vijaysri S, et al. Subversion of cell signaling pathways by hepatitis $\mathrm{C}$ virus nonstructural 5A protein via interaction with Grb2 and P85 phosphatidylinositol 3-kinase. J Virol 2002; 76: 9207-17. 